\title{
Assessing Asymmetries In Change Of Direction Speed Performance; Application Of Change Of Direction Deficit
}

Original Research

Funding Statement: No external funding was received for this work.

Conflict of Interest: There are no conflicts of interest concerning this paper

Thomas Dos'Santos ${ }^{1 \#}$, Christopher Thomas ${ }^{1}$, Paul A Jones ${ }^{1}$ and Paul Comfort ${ }^{1}$

${ }^{1}$ Human Performance Laboratory, Directorate of Sport, Exercise, and Physiotherapy,

University of Salford, Greater Manchester, United Kingdom

\#Corresponding Author: Thomas Dos'Santos

Telephone: +447961744517

Email: t.dossantos@edu.salford.ac.uk

Preferred running head: Change of direction deficit asymmetry

Abstract word count: 288 words

Manuscript word count: 4276 words

Number of tables and figures: 3 Tables and 3 Figures 


\section{Abstract}

The aims of this study were to quantify asymmetries in change of direction (COD) performance via completion time and COD deficit, and determine its influence on asymmetry profiling of COD ability. A secondary aim was to evaluate the relationship between linear speed, 505 time and COD deficit. Forty-three youth netball athletes (age: $15.4 \pm 1.1$ years, height: $1.71 \pm 0.06 \mathrm{~m}$, mass: $63.3 \pm 6.6 \mathrm{~kg}$ ) performed the 505 for both left and right limbs and a $10 \mathrm{~m}$ sprint test. Asymmetries in 505 completion time and COD deficit were quantified for dominant (D) (faster) and non-dominant (ND) (slower) directions. Paired sample t-tests revealed significant differences between D and ND directions for 505 time and COD deficit $(p<0.0001, g=-0.53$ to -0.60$)$. Substantially greater asymmetries for COD deficit were observed compared to 505 time $(p<0.0001, g=1.03$ ). Only two subjects displayed an asymmetry $\geq 10 \%$ based on 505 times. Conversely, based on COD deficit, 21 subjects demonstrated asymmetries $\geq 10 \%$. Large significant associations were observed between 505 time and COD deficit $(r=0.500-0.593, p \leq 0.002)$. Large significant inverse associations were demonstrated between $10 \mathrm{~m}$ sprint time and COD deficit $(r=-0.539$ to $-0.633, p \leq$ 0.001) indicating faster athletes had longer COD deficits. Nine subjects were classified differently for COD ability when comparing standardized scores for 505 time versus COD deficit. Quantification of asymmetries in COD ability should be based on COD deficits; inspection of 505 times only could lead to misinterpretations of an athlete's COD symmetry and COD ability. Faster youth netball athletes demonstrate longer COD deficits, thus, researchers and practitioners are encouraged to improve their youth netball athletes' ability to rapidly decelerate, change direction and reaccelerate from $180^{\circ}$ turns.

Key words: performance deficits; 505 test; limb dominance; maneuverability; netball; sprint 


\section{INTRODUCTION}

The ability to change direction efficiently is a key movement linked to decisive actions in multidirectional sport $(3,7,16,21,30)$ and change of direction (COD) speed provides the mechanical and physical basis underpinning agility (22). Single COD tests such as the 505 and modified $505(\bmod 505)$ are commonly used to examine COD speed in various athletic populations $(6,9,22-24,29,31)$. However, these tests are confounded by linear speed $(18$, $22,23,27)$ as only $31 \%$ of the time during the 505 is spent actually changing direction $(23)$. A method which eliminates this limitation is the examination of the COD deficit (CODD), whereby an athlete's $10 \mathrm{~m}$ or yard sprint time is subtracted from the 505 time or alternative $10 \mathrm{~m}$ COD sprint test $(22,23,26)$. The application of CODD has been suggested to provide a more isolated measure of COD ability which is not influenced by an athlete's acceleration and linear speed qualities $(18,22,23)$.

Being equally proficient and fast at changing direction from both limbs and directions would be advantageous from a performance perspective given the unpredictable nature of multidirectional sports. Researchers have attempted to examine asymmetries in completion time between directions (push off limbs) during COD speed tests, observing significant biases and faster performance from a push off limb or direction $(5,10,25)$. For example, Hart et al. (10) reported a performance deficit of 5-10\% ( 0.72 seconds, $p<0.001)$ between dominant (D) (fastest) and non-dominant (ND) (slower) directions of travel during the preplanned multidirectional AFL agility test in Australian Rules football players. Rouissi et al. (25) demonstrated no significant differences between completion times of the modified Illinois COD test and the mirrored inverted equivalent of this test when analyzed as pooled data $(p>0.05$, effect size $[E S]=0.05)$ in 46 soccer players. However, when inspecting the individual responses 24 players $(52 \%)$ had a significantly better time during the inverted modified Illinois COD test compared to the modified Illinois COD test. However, it is worth 
noting that the AFL and modified Illinois COD tests involve five to seven CODs, and have long test durations and distances of 7.5-12 seconds and 30-40 m, respectively. Thus, these tests are heavily influenced by linear speed qualities and therefore, may not provide a valid indication of $\mathrm{COD}$ ability $(22,23,27,32,33)$, but a more suitable assessment of maneuverability (21).

Asymmetries in completion times of single COD tests $\left(\bmod 505\right.$ and $90^{\circ}$ cut) have also been investigated with significant differences reported between D and ND directions $(p<0.0001$, $g=-0.49$ to -0.61$)$ resulting in mean imbalances of $2.94 \pm 1.98 \%$ and $4.24 \pm 3.28 \%$, respectively (5). The imbalances observed are not greater than the $10-15 \%$ threshold which has been stated to represent a potentially problematic asymmetry (15), however the low imbalances could be explained by not applying CODD; a more isolated measure of COD ability (21). Physical qualities such as acceleration and linear speed could mask deficiencies in COD ability when only examining asymmetries in completion time $(22,23,27)$. Furthermore, inspection of completion time and CODD can lead to different evaluations regarding athletes an COD ability (22). This is a concern as practitioners use test results to inform the decisions they make regarding training prescription and asymmetry profiling.

To date, no study has comprehensively quantified asymmetries in COD ability using the CODD method, whereby larger imbalances between limbs are likely to be demonstrated than solely comparing completion time between directions. This is of great interest to practitioners as large imbalances could indicate a deficiency in COD ability to a specific direction which can then be used to subsequently inform future training. Nimphius et al. (22) classified male cricketers 505 and CODD into preferred and non-preferred sides, but did not quantify asymmetries between directions. Rouissi et al. (26) reported significant differences $(p<0.01$, $\mathrm{ES}=0.30-40)$ in $45^{\circ}, 90^{\circ}, 135^{\circ}$ and $180^{\circ} 10 \mathrm{~m}$ COD completion time and CODDs between directions based on limb preference. 
However, the authors failed to compare completion times and CODDs based on directional dominance, which theoretically would have demonstrated greater differences. Consequently, further research is required quantifying asymmetries between faster and slower directions of travel in COD performance, through the application of the CODD and to examine its influence on asymmetry profiling of COD ability.

Therefore, this study investigated asymmetries in 505 COD speed performance in youth netball players because of the frequency of $180^{\circ}$ turns in netball (30) and reliability of this assessment within this population (2). The aims of this study were to quantify asymmetries in COD performance via completion time and CODD, and to determine its influence on asymmetry profiling of COD ability. A secondary aim was to evaluate the relationship between linear speed, COD time measured by the 505 and CODD. It was hypothesized that significant differences would be demonstrated between D and ND 505 times and CODDs, with greater asymmetries (percentage imbalances) observed in CODDs compared to completion time. It was also hypothesized that faster 505 times would be associated with shorter CODDs, and linear speed would demonstrate significant relationships with 505 time but not CODD.

\section{METHODS}

\section{Experimental Approach to the problem}

This study investigated between direction asymmetries in CODD and completion time as measured by the 505 in youth netball athletes over one testing session. A cross sectional analysis was conducted, and paired sample t-tests were used to compare D and ND 505 completion times and CODDs to determine if significant differences between directions were present. The plant foot which produced the fastest 505 time was defined as D COD speed performance. 
Percentage imbalances between D and ND performance (asymmetry index) were also quantified and compared between 505 completion time and CODD to explore which method produces greater asymmetries; and to evaluate the subsequent effect on asymmetry classification. Pearson's correlations were used to determine the relationship between 505 times, $10 \mathrm{~m}$ sprint times and CODD for D and ND performance. Standardized Z scores for 505 times and CODDs for D and ND performance were implemented to determine whether scores for each subject were meaningfully different from the group mean and to inspect if different classifications of COD ability were present.

\section{$\underline{\text { Subjects }}$}

Forty-three female youth netball players (age: $15.4 \pm 1.1$ years, height: $1.71 \pm 0.06 \mathrm{~m}$, mass: $63.3 \pm 6.6 \mathrm{~kg}$, maturity offset: $2.9 \pm 0.8$ years post peak height velocity) from a regional performance academy participated in this study. Based on the work of Dos'Santos et al. (5) for D versus ND differences in mod505 completion time, a minimum sample size of 31 was determined from an a priori power analysis using $\mathrm{G}^{*}$ Power (Version 3.1, University of Dusseldorf, Germany) (8) based upon an effect size of 0.61 , a power of 0.95 and type 1 error or alpha level of 0.05 . The investigation was approved by the institutional ethics review board, and all subjects were informed of the benefits and risks of the investigation prior to signing an institutionally approved consent form to participate in the study, with consent from the parent or guardian of all players under the age of 18 .

Testing was conducted midseason during which time all subjects were training with sessions comprising all the elements of performance including 4-5 netball-specific training sessions, plus two resistance training sessions each week. At the time of testing, subjects were at the end of a 4-week strength mesocycle. 
All subjects rested the day before testing and were asked to attend testing in a fed and hydrated state, similar to their normal practices before training. All subjects were familiar with the tests performed in this study as part of their normal training and monitoring regime.

\section{$\underline{\text { Procedures }}$}

On arrival, all subjects had their body mass (Seca Digital Scales, Model 707), standing and seated height (Stadiometer; Seca, Birmingham, United Kingdom) measured to the nearest 0.1 $\mathrm{kg}$ and $0.1 \mathrm{~cm}$, respectively, and used to estimate maturity offset (20). Prior to data collection, each subject performed a typical pre-game warm-up routine incorporating low-intensity jogging (10 minutes), sprint and low intensity plyometric drills (i.e., high knee marching, running, skipping over $20 \mathrm{~m})$, short sprint $(10 \mathrm{~m})$ and change of direction drills (180 ${ }^{\circ}$ turns). All testing was performed indoors on a hardwood netball court on the same day in the following order: $10 \mathrm{~m}$ sprint and 505 COD speed test.

\section{$\underline{\text { Sprint testing }}$}

The $10 \mathrm{~m}$ sprint test is a required test from the national governing body. Subjects performed two warm up trials from $10 \mathrm{~m}$ at $50 \%$ and $75 \%$ maximum effort before being instructed to give a maximal effort for the whole $10 \mathrm{~m}$. All subjects performed three maximum effort sprint trials, with two minutes' rest between trials, using single beam (accuracy to 1/1000th of a second) Brower photocell timing Gate (model number BRO001; Brower, Draper, UT, USA) setup at 0 - and $10 \mathrm{~m}$; time was recorded to the nearest 0.001 seconds. Timing gates were placed at the approximate hip height for all athletes as previously recommended (34), to ensure that only one body part such as the lower torso breaks the beam. Subjects started 0.5 $\mathrm{m}$ behind the first gate, to prevent any early triggering of the initial start gate, from a twopoint staggered start. The best performance from each of the three trials was used for further analysis. 


\section{$\underline{505 \text { change of direction speed test }}$}

Following the sprint testing subjects were provided five minutes rest before progressing to the COD speed test. Change of direction speed was assessed utilizing a 505 test (6) and each subject performed two warm up 505 trials (in each direction) from $15 \mathrm{~m}$ at $50 \%$ and $75 \%$ maximum effort before being instructed to give a maximal effort for the whole 505 . To quantify asymmetries each subject performed three maximum effort trials in each direction in an alternating order, turning with left and right foot plants, with two minutes' rest between each trial. Subjects started $0.5 \mathrm{~m}$ behind the same photocell gates described above, to prevent any early triggering of the initial start gate, from a two-point staggered start. Timing gates were again placed at the approximate hip height for all athletes. Subjects were instructed to sprint to a line marked $15 \mathrm{~m}$ from the start line, placing either left or right foot on the line (depending on the trial), turn $180^{\circ}$ and sprint back $5 \mathrm{~m}$ through the finish. If the subject changed direction before hitting the turning line, or turned off the incorrect foot, the trial was disregarded and the subject completed another trial after the rest period. 505 completion times for each trial was recorded to the nearest 0.001 seconds. The best of all three trials was determined for each direction and used for further analysis. CODD was calculated using the formula: 505 time - $10 \mathrm{~m}$ sprint time $(22,23)$.

\section{$\underline{\text { Asymmetry index }}$}

The plant foot (direction) with the fastest completion time was defined as D COD speed performance, whereas the slower side was the ND side $(5,10)$. Asymmetry index for D and ND COD speed performance was calculated by the formulae (dominant - non dominant / dominant $\mathrm{x}$ 100) (5). Asymmetry thresholds for 505 time and CODD were established using mean imbalance - $(0.2 S D$ of the mean $)(5,17)$. 
Subjects with imbalances which exceeded the threshold were classified as asymmetrical, imbalances below the threshold were subsequently classified as balanced $(5,17)$.

\section{$\underline{\text { Statistical Analyses }}$}

All statistical analyses were performed using SPSS (version 23, IBM, New York, NY, USA) and Microsoft Excel (version 2016, Microsoft Corp., Redmond, WA, USA). Mean \pm SD were calculated for all variables. Normality was confirmed for all variables using a Shapiro Wilkstest. Within-session reliability was assessed with intraclass correlation coefficients (ICC) and coefficients of variation (CV) calculated as SD/mean x 100. Minimum acceptable reliability was determined with an ICC $>0.8$ and CV $<10 \%(1,4)$. Magnitude of differences between D and ND directions were assessed with paired sample t-tests and effect sizes calculated using Hedges' $g$ method (11) and were interpreted as trivial $(<0.19)$, small $(0.20-0.59)$, moderate $(0.60-1.19)$, large $(1.20-1.99)$, and very large $(2.0-4.0)(13)$. Relationships between sprint, 505 completion time and CODD were analysed using Pearson's product-moment correlation (with 95\% confidence intervals) and were Bonferroni corrected to control for type 1 error. The D and ND sides were examined separately and correlations were evaluated as follows: trivial $(0.0-0.09)$, small $(0.10-0.29)$, moderate $(0.30-0.49)$, large $(0.50-0.69)$, very large $(0.70-0.89)$, nearly perfect $(0.90-0.99)$, and perfect $(1.0)(12)$. The criterion for significance was set at $p \leq 0.05$. Additionally, utilizing a similar method to Nimphius et al. (22) to examine if 505 times and CODDs provided different indications of COD ability, Z scores were calculated by the formula: $\mathrm{Z}$ score $=($ subjects score - group mean $) / \mathrm{SD}$. Again, D and ND sides were examined separately. Worthwhile differences were also calculated (differences in z scores for 505 time and CODD) by the methods described by Nimphius et al. (22). 


\section{RESULTS}

High reliability and low variance were observed for $10 \mathrm{~m}$ sprint $(\mathrm{ICC}=0.905, \mathrm{CV}=2.2 \%)$, 505 left $(\mathrm{ICC}=0.837, \mathrm{CV}=2.3 \%)$ and 505 right performance $(\mathrm{ICC}=0.876, \mathrm{CV}=2.3 \%)$; all meeting minimum acceptable reliability criteria. Descriptive statistics and comparisons between D and ND directions for all tests are presented in Table 1. Moderate and significant differences were observed between D and ND directions for 505 time $(p<0.0001, g=-0.60)$ and small significant differences between D and ND directions for CODD $(p<0.0001, g=-$ 0.53) were demonstrated. Figure 1 illustrates the individual asymmetries for 505 time and CODD with positive values indicating right limb turning advantage and negative values indicating a left limb turning advantage. Based on the asymmetry thresholds of $-2.8 \%$ for 505 time, 13 of 43 subjects (30\%) were classified as asymmetrical, although only two subjects (5\%) demonstrated asymmetries greater than $10 \%$ (Figure 1). Conversely, significantly greater asymmetries $(p<0.0001, g=1.03)$ were observed for CODD compared to 505 time. 15 of 43 subjects (35\%) were classified as asymmetrical based on the asymmetry threshold of $-14.5 \%$ for CODD and 21 subjects (49\%) exhibited asymmetries greater than $10 \%$ (Figure 1).



The correlation data with $95 \%$ confidence intervals are presented for D COD performance in Table 2 and ND data in Table 3. Large significant positive associations between 505 time and CODD were observed for both D and ND COD performance $(r=0.500-0.593, p \leq 0.002)$. Moderate non-significant relationships were observed between $10 \mathrm{~m}$ sprint time and 505 time for $\mathrm{D}$ and ND COD performance $(r=0.355-0.359, \mathrm{p} \geq 0.054)$. However, large significant inverse relationships were demonstrated between $10 \mathrm{~m}$ sprint time and CODD $(r=-0.539$ to 0.633, $p \leq 0.001)$ indicating faster athletes had longer CODDs. 


$$
\begin{aligned}
& \text { **Insert Table } 2 \text { around here** } \\
& \text { **Insert Table } 3 \text { around here** }
\end{aligned}
$$

Figure 2 illustrates the $\mathrm{Z}$ scores for 505 completion time and CODD for each subject. For the D direction 9 of 43 subjects (21\%) (subject 7, 9, 13, 15, 19, 26, 33, 36 and 39) had a 505 time and CODD suggesting opposite COD ability (i.e., the $\mathrm{Z}$ score shifted from positive for 505 time (slower than average) to negative for CODD (faster than average) or vice versa. Similarly, for the ND direction 9 of 43 subjects (21\%) (subject 1, 4, 5, 7, 9, 15, 19, 27 and 35) also had a 505 time and CODD suggesting opposite COD ability.

$$
\text { **Insert Figure } 2 \text { around here** }
$$

Figure 3 displays the differences in $\mathrm{Z}$ scores (CODD - 505 time) for $\mathrm{D}$ and ND COD performance for each subject. For the D direction, there was a worthwhile difference for 32 of 43 subjects (74\%) indicating they had their COD ability overestimated or underestimated. A similar trend was present for the ND direction, with 34 of 43 subjects (79\%) having their COD ability overestimated or underestimated.

*Insert Figure 3 around here**

\section{DISCUSSION}

The primary findings from this study were COD dominance was demonstrated by netball athletes, with significant differences observed between D and ND directions for 505 time and CODD $(p<0.0001, g=-0.53$ to -0.60$)$. However, significantly greater percentage imbalances, thus asymmetries were demonstrated in CODDs compared to 505 times $(p<$ 0.0001, $g=1.03$, Table 1) which subsequently impacted the profiling and classification of athletes' COD symmetry (Figure 1). Thus, the application of CODD to quantify asymmetries 
in COD performance appears to be a suitable method in comparison to 505 times. In agreement with the hypothesis, large positive associations were demonstrated between 505 time and CODD $(r=0.500-0.593, p \leq 0.002)$. However, large inverse relationships were observed between $10 \mathrm{~m}$ sprint and CODD for both D and ND directions $(r=-0.539-0.633, p$ $\leq 0.001$ ), indicating faster netball athletes demonstrated longer CODDs and therefore, may not have the capabilities to rapidly decelerate, turn and reaccelerate from high entry velocity $180^{\circ}$ turns.

Significant differences between D and ND directions for 505 time and CODD were observed ( $p<0.0001, g=-0.53$ to -0.60 ), which corroborates the findings of previous research that have reported asymmetries in the AFL agility test (10), modified Illinois COD test (25) and mod505 and $90^{\circ}$ cut completion time (5). However, substantially greater percentage imbalances were demonstrated in CODDs between directions (-11.9\% vs $-2.3 \%)$ compared to 505 times $(p<0.0001, g=1.03)$, which subsequently influenced COD asymmetry profiling (Figure 1). This discrepancy could be attributed to the CODD providing a more isolated measure of COD ability, whereas $69 \%$ completion time during 505 is comprised of linear running and therefore, imbalances in COD completion time will be diminished due to the influence of linear speed (23).

A between limb asymmetry greater than $10-15 \%$ has been suggested to represent potentially problematic asymmetry (15). With this in mind, only two of 43 subjects (5\%) demonstrated asymmetries greater than $10 \%$ based on 505 times (Figure 1). However, when asymmetries were evaluated for CODD a large proportion of subjects were reclassified, with 21 subjects (49\%) exhibiting asymmetries greater than $10 \%$, while 15 subjects were classified as asymmetrical exceeding the $-14.5 \%$ asymmetry threshold calculated in the present study (5, 17), respectively (Figure 1). 
Failure to inspect asymmetries in CODD and solely comparing 505 completion times between directions could lead to misinterpretations of an athlete's symmetry in COD ability, as a consequence of the notably lower asymmetries produced (Table 1, Figure 1). This result is problematic as practitioners use test results to identify strengths and weaknesses in their athletes to subsequently inform the prescription of future training. Resultantly, researchers and practitioners are encouraged to evaluate asymmetries in COD ability via the application of CODD and avoid interpretation of athletes' asymmetry in COD ability based on completion time.

To our knowledge this is the first study to quantify asymmetries in CODD during the 505 in an athletic population, reporting mean imbalances of $-11.9 \pm 12.8 \%$ between $\mathrm{D}$ and ND directions (Table 1). Based on the means presented in previous research, Nimphius et al. (22) would have reported a $-8.6 \%$ imbalance to the faster side in male cricketers CODDs. Although not quantified in their study, Rouissi et al. (26) would have found percentage imbalances of $-13.3 \%,-5.3 \%,-4.5 \%$ and $-4.5 \%$ between limbs for $45^{\circ}, 90^{\circ}, 135^{\circ}$ and $180^{\circ}$ CODs sprint tests. These percentage differences are lower than the present study for $180^{\circ}$ turn performance however, this discrepancy could be attributed to Rouissi et al. (26) comparing between direction performance based on limb preference, which may not necessarily be the push off limb equating to superior COD performance, as shown by Dos'Santos et al. (5). Thus, it could be speculated that greater imbalances may have been observed if asymmetries in COD performance were calculated based on directional dominance rather than limb preference. Nevertheless, youth netball players with asymmetries greater than the asymmetry threshold $-14.5 \%$ can be considered asymmetrical in $180^{\circ} \mathrm{COD}$ ability. 
In agreement with previous research significant differences $(p<0.0001, g=-0.60)$ in completion time were observed between $\mathrm{D}$ and ND directions $(5,10,25)$. With respect to CODD, 21 netball athletes (49\%) exhibited asymmetries greater than 10\%, while 15 netball athletes were classified as asymmetrical exceeding the $-14.5 \%$ asymmetry threshold (Figure 1), respectively. Thus, CODD asymmetries could be interpreted as a deficiency in COD ability to one side, indicating that an athlete possesses a faster and slower side when changing direction $180^{\circ}$. This is undesirable for multidirectional sport athletes where it would be advantageous to be equally proficient changing direction from either limb or direction. The information attained by examining CODDs asymmetries can subsequently be used by practitioners to identify strengths and weaknesses, and inform future training and monitoring of COD ability. Furthermore, identification of excessive CODD asymmetries in specific athletes could encourage further analysis into the potential mechanisms and causes of inferior performance to one side through qualitative video technique analysis and muscle strength asymmetry assessments. Researchers and practitioners are recommended to examine both sides in their COD speed tests while applying the CODD to eliminate bias and permit the quantification of asymmetry in COD ability. Obtaining this information would require no extra effort or additional equipment because COD speed tests are regularly performed in both directions and $10 \mathrm{~m}$ sprint times are commonly included in the testing batteries of various sports $(9,24,31)$.

Although it was not the aim of this study to determine the causes of asymmetries in COD performance, joint-joint coordination on approach and reacceleration leading to technical differences between sides or differences in braking strategies between D and ND sides could be implicated. Further research is required to understand the technical differences in COD ability from either limb via 3D motion analysis. Furthermore, the mixed evidence $(5,19,26$, 35) of whether muscle strength qualities impact on COD speed from either limb requires 
further research involving biomechanical analysis to identify the impact of strength asymmetries on approach, plant and re-acceleration characteristics.

Consistent with the findings of previous studies $(18,22,23)$, athletes which demonstrated smaller CODDs produced faster 505 times $(r=0.500-0.593, p \leq 0.002)$. However, interestingly large significant inverse relationships were demonstrated between $10 \mathrm{~m}$ sprint time and CODD for D and ND performances ( $r=-0.539$ to $-0.633, p \leq 0.001)$, indicating faster youth netball athletes had longer CODDs and may not have the capacity to efficiently decelerate, change direction $180^{\circ}$ and reaccelerate. The youth female netballers may have been deficient in eccentric strength capacity which is fundamental for the deceleration requirements involved in $180^{\circ}$ turns $(14,28,29)$, especially with higher approach velocities. Therefore, this may justify why faster athletes demonstrated longer CODDs, compared to experienced male cricketers $(22)$ and American footballers $(18,23)$ in previous research, which observed no influence of linear speed on CODD. As such, further research is required investigating physical strength determinants of CODD in athletic populations.

The calculated $\mathrm{Z}$ scores for CODD and 505 time revealed 9 of 43 subjects (21\%) for D and ND COD demonstrated opposing COD abilities (i.e. a faster 505 times, but worse CODD, or vice versa) (Figure 2). Worthwhile differences for a large proportion of subjects between the 505 time and CODD for D and ND COD performance were demonstrated with Figure 3 illustrating overestimations or underestimations in COD ability. These findings are supported by Nimphius et al. (22) who reported five of 17 cricketers displayed opposing COD abilities when comparing 505 and CODD Z scores. Furthermore Nimphius et al. (22) reported worthwhile differences between 505 and CODD in $94 \%$ and $88 \%$ of population for preferred and non-preferred sides. The results from the present study support the concept that CODD should be implemented for a more isolated measure of COD ability, whereby failure to 
inspect CODD from the 505 could lead to misinterpretations of an athlete's COD ability and could lead to incorrect COD training prescription (Figure 2) (22).

The results from the present study should be interpreted with respect to the sex and age of the population, and the angle of COD test. Further research is required quantifying asymmetries in CODD utilizing different angled COD tests such as $45^{\circ}, 90^{\circ}$ and $135^{\circ}$, and comparing asymmetries from different athletic populations with considerations for sex and age. Furthermore, the results of this study are only representative of the time testing took place (mid-season) and therefore, may fluctuate at different points throughout the training year. Thus, further research comparing the sizes and directions of asymmetries throughout the training year is required.

\section{PRACTICAL APPLICATIONS}

Researchers and practitioners are encouraged to perform COD speed tests from both limbs to establish directional dominance and COD asymmetries in their athletes. Quantifying asymmetries based on COD completion time produces substantially lower percentage imbalances, which could mask deficiencies in COD ability, potentially leading to misinterpretations of an athlete's symmetry in COD ability. Consequently, the quantification and profiling of an athlete's asymmetry in COD ability should be based on CODD, due to the substantially greater percentage imbalances observed, and ability to identify a greater proportion of athletes demonstrating imbalances greater than $10-15 \%$. Youth netball athletes demonstrating CODD asymmetries $\geq 14.5 \%$ during the 505 can be considered asymmetrical. Faster youth netball athletes demonstrate longer CODDs and therefore, practitioners are encouraged to improve their youth netball athlete's ability to rapidly decelerate, change direction and reaccelerate from the $180^{\circ}$ directional change. Assessing COD ability using the CODD provides practitioners with an isolated measure of COD ability, and appears to be a 
more suitable method for quantifying asymmetries in athletic populations to inform future

training. When performing test batteries, sprint and COD assessments should be performed in

the same session to avoid between session differences in running speed effecting the assessment of CODD.

\section{REFERENCES}

1. Atkinson $G$ and Nevill AM. Statistical methods for assessing measurement error (reliability) in variables relevant to sports medicine. Sports Medicine 26: 217-238, 1998.

2. Barber OR, Thomas C, Jones PA, McMahon JJ, and Comfort P. Reliability of the 505 Change of Direction Test in Netball Players. International journal of sports physiology and performance 3: 377-380, 2016.

3. Bloomfield J, Polman R, and Donoghue P. Physical demands of different positions in FA Premier League soccer. Journal of Sports Science and Medicine 6: 63-70, 2007.

4. Cortina JM. What is coefficient alpha? An examination of theory and applications. Journal of applied psychology 78: 98, 1993.

5. Dos'Santos T, Thomas C, Jones PA, and Comfort P. Asymmetries in single and triple hop are not detrimental to change of direction speed. Journal of Trainology 6: 35-41, 2017.

6. Draper JA and Lancaster MG. The 505 test: A test for agility in the horizontal plane. Australian Journal of Science and Medicine in Sport 17: 15-18, 1985.

7. Faude $\mathrm{O}$, Koch $\mathrm{T}$, and Meyer T. Straight sprinting is the most frequent action in goal situations in professional football. Journal of sports sciences 30: 625-631, 2012.

8. Faul F, Erdfelder E, Buchner A, and Lang A-G. Statistical power analyses using G* Power 3.1: Tests for correlation and regression analyses. Behav Res Methods 41: 1149-1160, 2009.

9. Gabbett TJ, Kelly JN, and Sheppard JM. Speed, change of direction speed, and reactive agility of rugby league players. The Journal of Strength \& Conditioning Research 22: 174-181, 2008.

10. Hart NH, Spiteri T, Lockie RG, Nimphius S, and Newton RU. Detecting deficits in change of direction performance using the preplanned multidirectional Australian Football League agility test. The Journal of Strength \& Conditioning Research 28: 3552-3556, 2014.

11. Hedges L and Olkin I. Statistical Methods for Meta-Analysis. New York: Acdemic Press, 1985.

12. Hopkins WG. Measures of reliability in sports medicine and science. Sports Medicine 30: 115, 2000.

13. Hopkins WG. A scale of magnitudes for effect statistics. A new view of statistics, 2002.

14. Jones P, Thomas C, Dos'Santos T, McMahon J, and Graham-Smith P. The Role of Eccentric Strength in $180^{\circ}$ Turns in Female Soccer Players. Sports 5: 42, 2017.

15. Kannus $P$, Isokinetic evaluation of muscular performance: implications for muscle testing and rehabilitation. International journal of sports medicine 15: S11-18, 1994.

16. Karcher $\mathrm{C}$ and Buchheit M. On-court demands of elite handball, with special reference to playing positions. Sports Medicine 44: 797-814, 2014.

17. Lockie RG, Callaghan SJ, Berry SP, Cooke ER, Jordan CA, Luczo TM, and Jeffriess MD. Relationship between unilateral jumping ability and asymmetry on multidirectional speed in team-sport athletes. The Journal of Strength \& Conditioning Research 28: 3557-3566, 2014.

18. Lockie RG, Farzad J, Orjalo AJ, Giuliano DV, Moreno MR, and Wright GA. A Methodological Report: Adapting the 505 Change-of-Direction Speed Test Specific to American Football. The Journal of Strength \& Conditioning Research 31: 539-547, 2017.

19. Maloney SJ, Richards J, Nixon DG, Harvey LJ, and Fletcher IM. Do stiffness and asymmetries predict change of direction performance? Journal of sports sciences 35: 547-556, 2016. 
20. Mirwald RL, Baxter-Jones ADG, Bailey DA, and Beunen GP. An assessment of maturity from anthropometric measurements. Medicine \& Science in Sports \& Exercise 34: 689-694, 2002.

21. Nimphius S. Increasing Agility. High-Performance Training for SportsChampaign, IL Human Kinetics, 2014.

22. Nimphius S, Callaghan SJ, Sptieri T, and Lockie RG. Change of direction deficit: A more isolated measure of change of direction performance than total 505 time. The Journal of Strength \& Conditioning Research 30: 3024-3032, 2016.

23. Nimphius S, Geib G, Spiteri T, and Carlisle D. " Change of direction deficit" measurement in Division I American football players. J Aust Strength Cond 21: 115-117, 2013.

24. Nimphius S, McGuigan MR, and Newton RU. Relationship between strength, power, speed, and change of direction performance of female softball players. The Journal of Strength \& Conditioning Research 24: 885-895, 2010.

25. Rouissi M, Chtara M, Berriri A, Owen A, and Chamari K. Asymmetry of the Modified Illinois Change of Direction Test Impacts Young Elite Soccer Players Performance. Asian journal of sports medicine 7, 2016.

26. Rouissi M, Chtara M, Owen A, Chaalali A, Chaouachi A, Gabbett T, and Chamari K. Effect of leg dominance on change of direction ability amongst young elite soccer players. Journal of sports sciences 34: 542-548, 2016.

27. Sayers MGL. Influence of test distance on change of direction speed test results. The Journal of Strength \& Conditioning Research 29: 2412-2416, 2015.

28. Spiteri T, Newton RU, Binetti M, Hart NH, Sheppard JM, and Nimphius S. Mechanical determinants of faster change of direction and agility performance in female basketball athletes. The Journal of Strength \& Conditioning Research 28: 2205-2214, 2015.

29. Spiteri T, Nimphius S, Hart NH, Specos C, Sheppard JM, and Newton RU. The contribution of strength characteristics to change of direction and agility performance in female basketball athletes J Strength Cond Res 28: 2415-2423, 2014.

30. Sweeting AJ, Aughey RJ, Cormack SJ, and Morgan S. Discovering frequently recurring movement sequences in team-sport athlete spatiotemporal data. Journal of Sports Sciences: 1-7, 2017.

31. Thomas C, Ismail KT, Comfort P, Jones PA, and Dos'Santos T. Physical Profiles of Regional Academy Netball Players. Journal of Trainology 5: 30-37, 2016.

32. Vescovi JD and McGuigan MR. Relationships between sprinting, agility, and jump ability in female athletes. Journal of sports sciences 26: 97-107, 2008.

33. Watts D. A brief review on the role of maximal strength in change of direction speed. J Aust Strength Cond 23: 100-108, 2015.

34. Yeadon MR, Kato T, and Kerwin DG. Measuring running speed using photocells. Journal of sports sciences 17: 249-257, 1999.

35. Young WB, James $R$, and Montgomery I. Is muscle power related to running speed with changes of direction? Journal of Sports Medicine and Physical Fitness 42: 282-288, 2002. 
Table 1. Dominant versus non-dominant comparisons for 505 time and CODD

\begin{tabular}{|c|c|c|c|c|c|c|c|c|c|}
\hline & \multicolumn{2}{|c|}{$\mathrm{D}$} & \multicolumn{2}{|c|}{ ND } & \multicolumn{2}{|c|}{ Imbalance $(\%)$} & \multirow[b]{2}{*}{$p$} & \multirow[b]{2}{*}{$g$} & \multirow[b]{2}{*}{$\begin{array}{c}\text { Asymmetry } \\
\text { Threshold (\%) }\end{array}$} \\
\hline & Mean & $\mathrm{SD}$ & Mean & $\mathrm{SD}$ & Mean & $\mathrm{SD}$ & & & \\
\hline $505(\mathrm{~s})$ & 2.531 & 0.089 & 2.590 & 0.104 & -2.3 & 2.3 & $<0.0001$ & -0.60 & -2.81 \\
\hline CODD (s) & 0.524 & 0.107 & 0.586 & 0.115 & -11.9 & 12.8 & $<0.0001$ & -0.53 & -14.5 \\
\hline
\end{tabular}

Key: CODD = Change of direction deficit; D: Dominant; ND: Non-dominant 
Table 2. Correlations between 505 time, CODD and $10 \mathrm{~m}$ sprint time for D direction

\begin{tabular}{cccc}
\hline & D 505 vs D CODD & D 505 vs 10 m sprint & D CODD vs 10 m sprint \\
\hline$r$ & $0.500^{* *}$ & 0.355 & $-0.633^{* *}$ \\
$95 \%$ CI & 0.23 to 0.69 & 0.06 to 0.59 & -0.78 to 0.63 \\
$r^{2}$ & 0.25 & 0.13 & 0.40 \\
$p$ & 0.002 & 0.058 & $<0.001$ \\
\hline
\end{tabular}

Key: CODD = Change of direction deficit; D: Dominant; ND: Non-dominant; CI= Confidence Interval $* *=p<0.01$ 
Table 3. Correlations between 505 time, CODD and $10 \mathrm{~m}$ sprint time for ND direction

\begin{tabular}{cccc}
\hline & ND 505 vs ND CODD & ND 505 vs 10 m sprint & ND CODD vs 10 m sprint \\
\hline$r$ & $0.593^{* *}$ & 0.359 & $-0.539 * *$ \\
$95 \%$ CI & 0.36 to 0.76 & 0.07 to 0.6 & -0.72 to -0.28 \\
$r^{2}$ & 0.35 & 0.13 & 0.29 \\
$p$ & $<0.001$ & 0.054 & 0.001 \\
\hline
\end{tabular}

Key: CODD = Change of direction deficit; D: Dominant; ND: Non-dominant; CI= Confidence Interval $* *=\mathrm{p}<0.01$ 


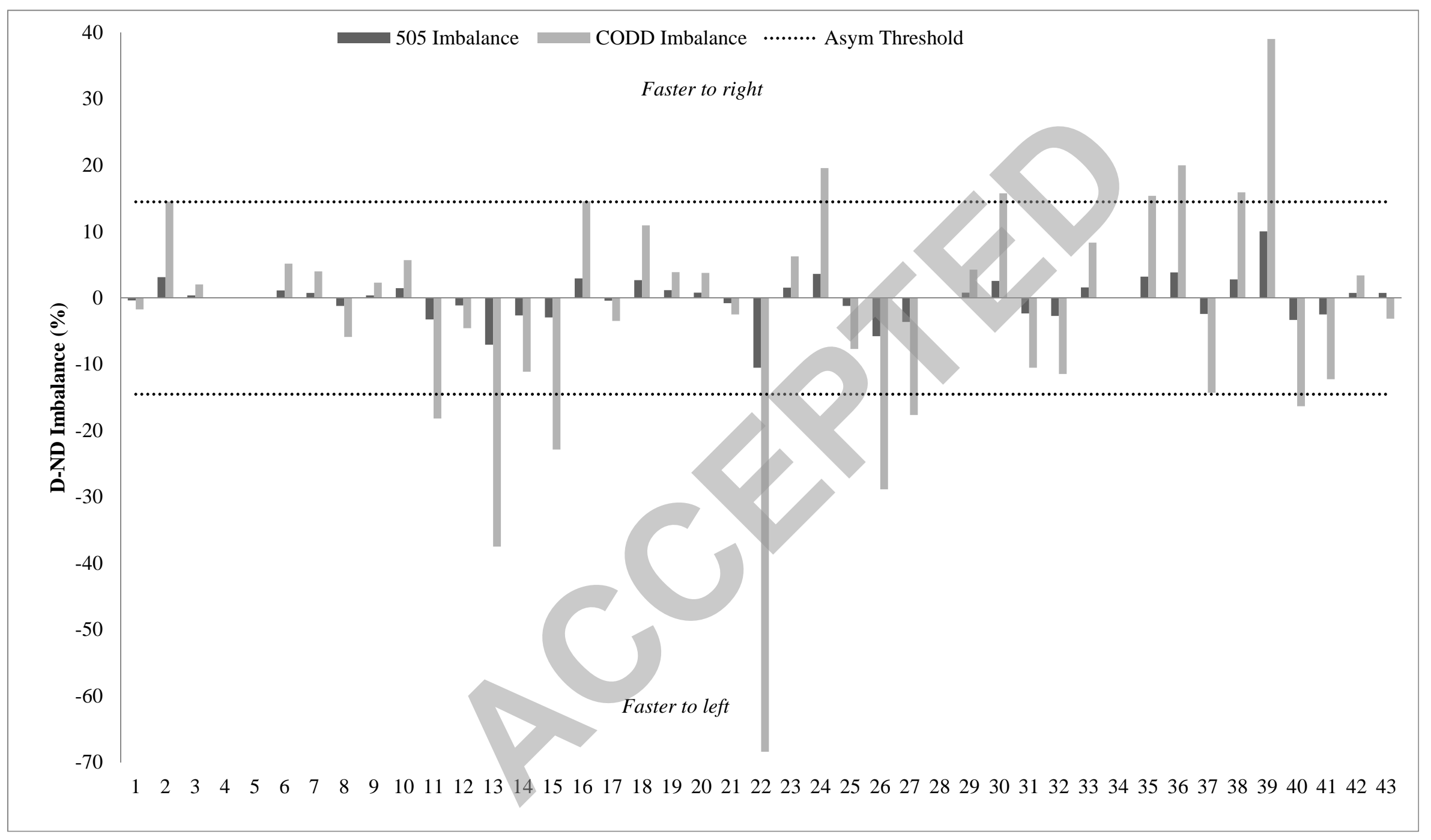

Figure 1. Individual imbalances in 505 times and CODDs (Positive values indicate right foot plant advantage. Negative values indicate left foot plant advantage 

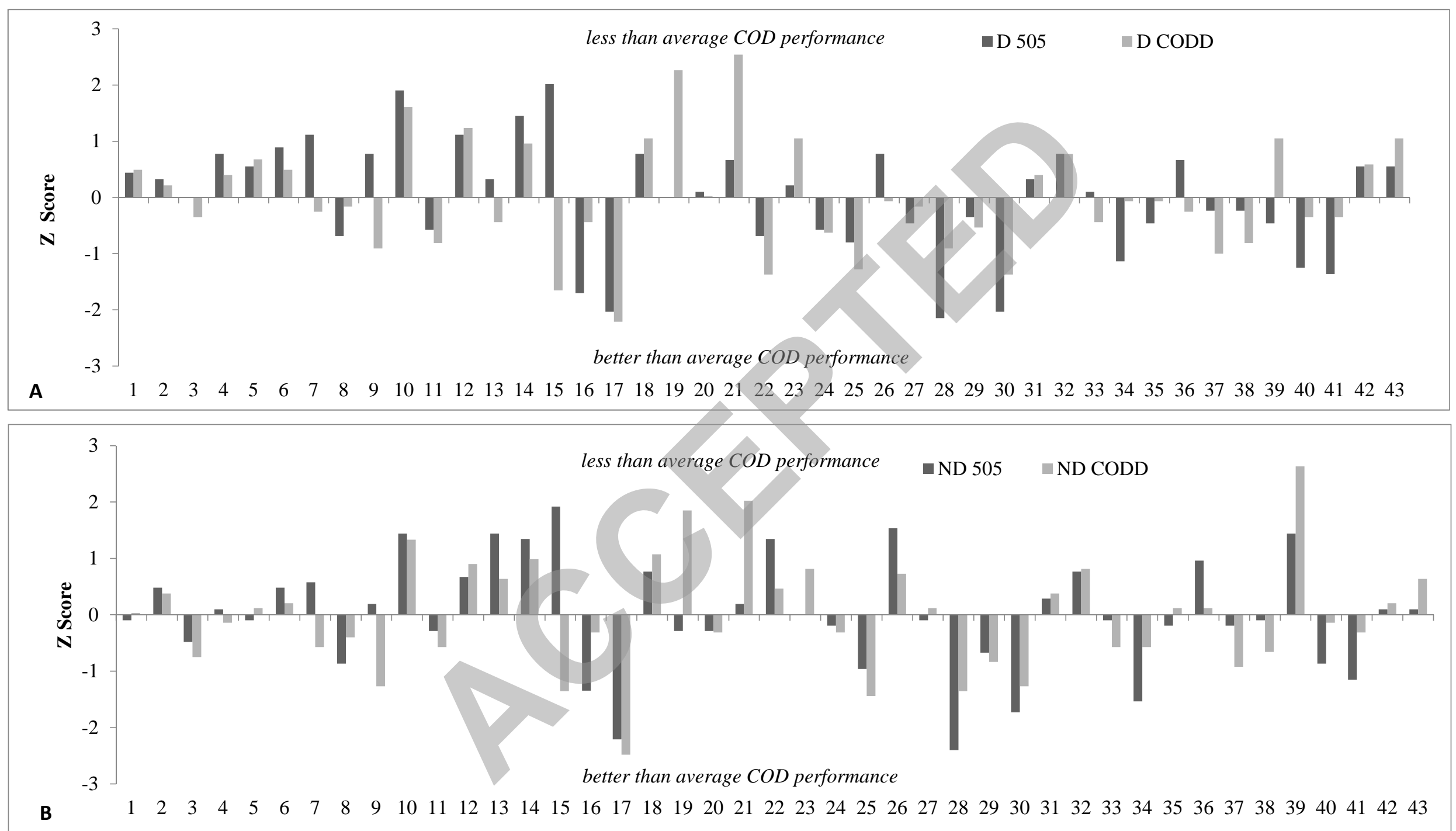

Figure $2 . \mathrm{Z}$ scores of the 505 time and CODD for each subject. $\mathrm{A}=\mathrm{D}$ direction results and $\mathrm{B}=\mathrm{ND}$ direction results. 

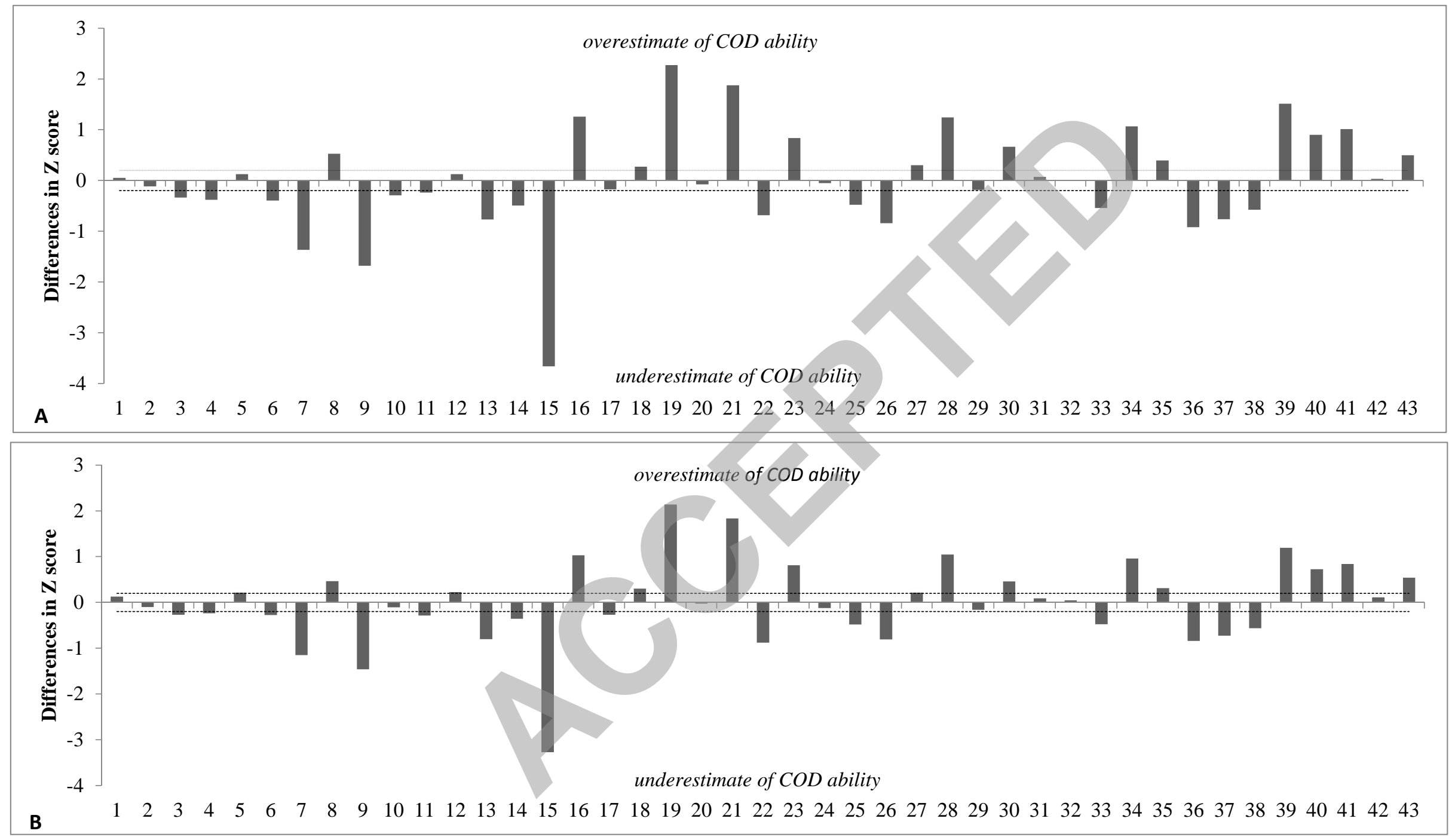

Figure 3. Differences in standardized scores (CODD -505 time) for each subject. $A=D$ direction results and $B=$ ND direction results. The smallest worthwhile change is represented by horizontal dotted lines. 